\title{
LA CIENCIA POLÍTICA EN GUATEMALA: EL RETO DE LA CONSOLIDACIÓN COMO DISCIPLINA INDEPENDIENTE*
}

\author{
DinORAH AZPURU \\ Wichita State University, Estados Unidos
}

\begin{abstract}
Resumen
La ciencia política surgió en Guatemala a finales de los años 60 y durante más de una década, tuvo que desarrollarse con las limitaciones impuestas por los regímenes autoritarios de turno. No obstante, desde su surgimiento las escuelas de ciencia política promovieron activamente la democratización del país. Al iniciarse el proceso democratizador a mediados de los 80 , se abrió el espectro de los temas de estudio e investigación. Algo similar ocurrió con el proceso de paz en los años 90 . A lo largo de tres décadas la disciplina ha contribuido en forma importante a la construcción de la democracia y la paz en Guatemala. Como disciplina, sin embargo, se ha visto limitada por los fuertes vínculos que ha mantenido con otras ciencias sociales y con el derecho. Durante 35 años los centros académicos que dan formación en ciencia política han enfrentado disyuntivas en cuanto al tipo de programas que deben ofrecerse a los estudiantes (grados técnicos, licenciatura o maestría), el perfil de los profesionales que egresan de los mismos vis-à-vis el mercado laboral y el énfasis en el estudio de la problemática guatemalteca vis-à-vis una perspectiva más global.
\end{abstract}

Abstract

The study of political science began in Guatemala in the late 1960s and for over a decade it had to develop with limitations imposed by the authoritarian regimes that governed the country. However, from the beginning the political science schools actively promoted democratization. When the democratization process began in the mid 1980s, the possibilities for research of different issues expanded. Something similar occurred with the peace process in the 1990s. Throughout three decades of existence, political science has significantly contributed to democracy-building and peace-building in Guatemala. However, as an academic discipline, it has been limited by the strong links that it has maintained with other social sciences and law. During 35 years the academic centers that teach political science have faced a series of dilemmas regarding the type of programs they offer to students (technical degree, bachelor degree or master degree), the profile of those who graduate vis-à-vis the job market and the emphasis in the study of the problems of Guatemala vis-à-vis a more global perspective.

\section{PALABRAS CLAVE • Ciencia Política • Guatemala • Democracia • Desarrollo Disciplinario}

\section{INTRODUCCIÓN}

Cuando dos universidades guatemaltecas iniciaron programas de estudio en el campo de la ciencia política a finales de los años 60 , el país se encontraba bajo el mando de gobiernos militares que pocos años después desencadenarían una de las campañas más sangrientas de represión en la historia reciente de América Latina. Guatemala era también, en ese momento, escenario de un

* La autora agradece la colaboración de diversas personas, en particular Carlos Escobar Armas y Karin Erbsen de Maldonado, quienes compartieron su amplia experiencia y brindaron ideas acerca del tema. 
enfrentamiento armado interno que llevó a una extrema polarización de la sociedad guatemalteca. En ese contexto, el desarrollo de la ciencia política hubo de adaptarse a las condiciones prevalecientes, tanto en términos de su objeto de estudio como en términos de la amplitud y apertura en el tratamiento de los temas de debate e investigación.

Hoy, 35 años después, y pese a múltiples problemas, la democracia y la paz prevalecen en el país y la ciencia política enfrenta nuevos retos orientados hacia la consolidación de ambas. A la vez, la disciplina enfrenta como disciplina académica, el reto de consolidar su independencia de otras ciencias sociales y expandir sus fronteras metodológicas y geográficas.

En ocasión del 25 aniversario de la Revista de Ciencia Política del Instituto de Ciencia Política de la Pontificia Universidad Católica de Chile se presenta la oportunidad de hacer un análisis de los aportes de la politología a la democratización de Guatemala en este período. En ese artículo se hace un breve recuento del desarrollo que ha tenido la disciplina en el país conforme han avanzado los procesos políticos, pero también se señalan los desafíos y problemas enfrentados a través de los años, y las perspectivas y retos de la disciplina en un futuro inmediato.

\section{EL SURGIMIENTO DE LA CIENCIA POLITICA EN GUATEMALA}

Podría parecer paradójico que la ciencia política surgiera precisamente a finales de los años 60 , en uno de los momentos históricos de mayor limitación a la libertad académica en Guatemala. Sin embargo, uno de los precursores de la disciplina en el país, el alemán Josef Thesing, expresa las razones:

La violencia y el terror determinaban el proceso político e impedían una apertura democrática y la modernización del país (...) pero también existían ciudadanos responsables en la política, la ciencia, la iglesia y en las universidades, dispuestos a buscar una salida a una situación aparentemente sin solución. Así surgió la idea de instaurar la asignatura de ciencias políticas. La adquisición de conocimientos políticos más amplios debía permitir una configuración más racional del debate político ${ }^{1}$.

Entre 1967 y 1968, Thesing presentó proyectos para iniciar un programa de estudios en ciencias políticas a los rectores de la universidad nacional, San Carlos (USAC) y de la universidad jesuita, Rafael Landívar (URL). Pese a cierta resistencia interna, ambas universidades aceptaron el reto. La Escuela de Ciencia Política de la USAC² inició labores en 1968, mientras que el Instituto de Ciencias Políticas y Sociales de la URL empezó a funcionar en $1970^{3}$.

En el contexto prevaleciente en ese momento en Guatemala, la academia en general era vista con sospecha por parte de los gobiernos autoritarios y los grupos conservadores del país, que enarbolaban la bandera del anticomunismo. En especial la USAC, era considerada por los grupos de poder como un "centro de formación intelectual para la guerrilla"4. La USAC fue en la década de los años 70 y principios de los 80 , objeto de indiscriminada represión por parte de los gobiernos militares y los grupos paramilitares.

Thesing, Josef (2000).

La Escuela de Ciencia Política de la USAC estaba adscrita a la Facultad de Derecho de esa casa de estudios.

El Instituto de Ciencias Políticas y Sociales de la URL surgió como unidad académica autónoma. Fue convertido en Facultad de Ciencias Políticas y Sociales a finales de los años 80.

$4 \quad$ Thesing, Josef (2000). 
Dicha realidad impuso obvias restricciones al desarrollo de la investigación y al debate que sobre temas políticos y sociales podía desarrollarse al interior de los salones de clase a través de los años. No obstante, ambas universidades buscaron espacios para promover el conocimiento y la discusión acerca de temas como la política, la democracia, los derechos humanos y la convivencia pacífica. Las escuelas de Ciencia Política iniciaron publicaciones regulares, siendo estas la Revista Política y Sociedad en la USAC y la Revista Estudios Sociales en la URL. Un momento determinante fue la realización en 1980 de un seminario llamado Realidad Nacional, organizado por el Instituto de Ciencias Políticas de la URL ${ }^{5}$, en el cual se discutieron abiertamente, aunque a un nivel muy superficial -dado el contexto prevaleciente-, temas como la violencia, las políticas sociales de vivienda y salud y la ética política. Fue éste el primer evento público en el que en muchos años se debatieron públicamente temas en ese entonces considerados políticamente sensibles ${ }^{6}$.

Todavía en un período de alta polarización ideológica en el país, debido en buena parte al conflicto armado que escaló en intensidad a principios de los años 80, surgió en 1983 el Instituto de Estudios Políticos y Relaciones Internacionales (EPRI) en la Universidad Francisco Marroquín (UFM), una universidad privada ubicada en la derecha del espectro político.

A lo largo de la década de los 80 y todavía en alguna medida en la década de los 90, las tres unidades académicas se identificaron cada una con una ideología política determinada -aunque no necesariamente todos los miembros del claustro de catedráticos y los estudiantes, sí una mayoría de los mismos-. Así, la Escuela de Ciencia Política de la USAC podía ser identificada con la línea social-demócrata; la Facultad de Ciencias Políticas y Sociales de la URL con la línea social-cristiana y el Instituto de Estudios Políticos y Relaciones Internacionales de la UFM con el liberalismo económico. Paralelamente, surgieron o se fortalecieron en el país centros de investigación académica que en una u otra forma se identificaban con determinadas universidades y captaban a sus egresados, en particular los provenientes de las escuelas de ciencia política. Los principales centros de investigación son la Facultad Latinoamericana de Ciencias Sociales (FLACSO), identificado con la USAC; la Asociación de Investigación y Estudios Sociales (ASIES), identificada con la URL; y el Centro de Investigaciones Económicas Nacionales (CIEN), identificado con la UFM. Otro centro de investigación que también incorporó desde un inicio egresados de ciencia política es el Instituto Centroamericano de Estudios Políticos (INCEP), cuya sede se encuentra en Guatemala ${ }^{7}$.

Diversos factores nacionales e internacionales confluyeron para dar lugar al proceso de liberalización política en el país a mediados de los años 80 , el cual concluyó con las elecciones presidenciales realizadas en 1985. La discusión acerca de cuándo inicia y cuándo termina la liberalización y la transición a la democracia en Guatemala va más allá del propósito de este artículo ${ }^{8}$, pero en todo caso, fue evidente que la intensidad del debate acerca de temas políticos se fue intensificando conforme se avanzaba en ambos procesos.

5 El II Seminario Permanente sobre la Realidad Nacional se realizó cinco años después, organizado por la Asociación de Investigación y Estudios Sociales (ASIES), y ha continuado hasta la fecha. En el año 2004 se realizó la XX Sesión Anual.

$6 \quad$ Estos y otros temas más sensibles ya estaban siendo abordados al interior de las unidades académicas de ciencia política y otros centros de estudio, pero prevalecía un ambiente de terror que limitaba las posibilidades de reunión y libre expresión.

7 El INCEP también puede ser considerado como un centro de ideología social-cristiana. En un inicio, el INCEP, ASIES y la URL recibieron importantes aportes económicos de la Fundación Konrad Adenauer de Alemania.

$8 \quad$ En este sentido es útil la conceptualización que hacen Juan Linz y Alfred Stepan de los términos de liberalización, transición y democratización. Ver Linz, J. y A. Stepan (1996). 
Los primeros indicios de este cambio se dieron en ocasión de la redacción de la nueva Constitución Política de la República por la Asamblea Nacional Constituyente que fue electa en elecciones libres en 1984. La nueva constitución entró en vigencia en mayo de 1985, habiéndose superado en la misma restricciones como la prohibición de participación de partidos políticos con ideología comunista; se introdujeron además temas novedosos, entre ellos el reconocimiento de Guatemala como un Estado multicultural y la creación de instituciones como el Procurador de Derechos Humanos y la Corte de Constitucionalidad. Puede asegurarse que muchos de esos temas fueron llevados a la mesa de discusión de la Asamblea Constituyente por académicos y grupos de la sociedad civil que recién empezaba a reorganizarse luego del período de intensa represión.

\section{LA NUEVA AGENDA: LOS DESAFÍOS DE LA DEMOCRATIZACIÓN Y LA CONSTRUCCIÓN DE LA PAZ}

Con el proceso de democratización iniciado a partir de 1985 se abrieron nuevas posibilidades de investigación y debate relacionadas con la construcción del nuevo régimen democrático. En las unidades académicas de ciencia política y los centros de investigación antes señalados, el énfasis en los primeros años fue puesto en la construcción de la institucionalidad democrática, la legislación necesaria para dar soporte al nuevo régimen y el rol de los partidos políticos. Temas como la participación ciudadana y los movimientos sociales también fueron abordados, pero con mayor cautela ${ }^{9}$. Durante los primeros años del proceso democratizador el debate público de ciertos temas como la subordinación del ejército al poder civil, los derechos de los pueblos indígenas y la tenencia de la tierra todavía continuó sujeto a limitaciones, pero la discusión a nivel académico se intensificóno ${ }^{10}$.

Con el proceso de negociaciones de paz (1991-1996) se escribe un nuevo capítulo en la historia política de Guatemala, dándose paralelamente una apertura sin precedentes a las posibilidades de investigación política y social y al debate público de temáticas hasta entonces consideradas como tabú. La academia entró a formar parte activa del proceso de negociaciones de paz a través de su participación activa en la llamada Asamblea de la Sociedad Civil (ASC), la cual agrupaba a las principales organizaciones sociales en el país. La ASC presentó numerosas propuestas a la mesa de negociaciones de paz en la cual los dos actores principales eran el gobierno guatemalteco y la guerrilla unificada en la Unidad Revolucionaria Nacional Guatemalteca (URNG). La amplitud de los temas discutidos en las negociaciones de paz hizo necesario hacer acopio de estudios e investigaciones realizadas por la comunidad académica, incluyendo la ciencia política ${ }^{11}$. El acuerdo final para una paz firme y duradera, firmado en diciembre de 1996, refleja esa realidad.

9 Por ejemplo en el Congreso Centroamericano de Sociología realizado en Guatemala en 1988 se abordaron temas de índole social. Las escuelas de ciencia política de la USAC y la URL patrocinaron conjuntamente la organización de este Congreso, lo cual dio lugar a un acercamiento entre los estudiantes y egresados de ambas.

10 La profundización de ciertos temas todavía implicaba riesgos para los investigadores sociales. En septiembre de 1990 la antropóloga Myrna Mack fue asesinada por sus investigaciones acerca de poblaciones desplazadas en áreas de conflicto.

11 Cabe recordar que los acuerdos de paz en Guatemala han sido considerados a nivel internacional como acuerdos de segunda generación, que incluyen además del cese al fuego, una multiplicidad de temas. Además de los acuerdos operativos, resaltan los acuerdos sustantivos acerca del tema de derechos humanos, el acuerdo sobre identidad y derechos de los pueblos indígenas, el acuerdo sobre aspectos socioeconómicos y situación agraria, el acuerdo sobre el fortalecimiento del poder civil y el rol de las fuerzas armadas en una sociedad democrática y el acuerdo sobre reformas constitucionales y régimen electoral. Ver Pásara, Luis (2003). 
Uno de los mayores retos para la ciencia política desde la época de las negociaciones de paz, es el tratamiento de la realidad multicultural de Guatemala. Con los acuerdos de paz se inició un proceso irreversible de demanda de mayor participación de la población indígena del país, que representa alrededor de la mitad del total de guatemaltecos. En el país se ha establecido un modelo de democracia liberal occidental, que ha sido criticado por algunos académicos mayas (en especial antropólogos), quienes han señalado que el sistema político debe tener mayor apertura y creatividad para reflejar la realidad multicultural del país. La disciplina del derecho ha abordado esta problemática analizando lo que se ha llamado "el derecho consuetudinario". Sin embargo, la ciencia política no ha ofrecido, hasta el momento, muchas luces en la búsqueda de alternativas para adaptar la "política" a la realidad multiétnica y multicultural del país. Al igual que en otras ramas del conocimiento, todavía existen muy pocos politólogos Mayas, pero el tema de la política y la multiculturalidad no debe ser únicamente objeto de investigación de politólogos Mayas, sino de todos aquellos interesados en el desarrollo político del país.

\section{DISYUNTIVAS DE LOS PROGRAMAS DE CIENCIA POLÍTICA}

A lo largo de tres décadas las escuelas de ciencia política en Guatemala han enfrentado diversos dilemas en cuanto a la formación de estudiantes. Aquí se discuten algunos de ellos.

El primer dilema tiene relación el grado académico que se debe ofrecer a los estudiantes: ¿un grado técnico, un pregrado, una licenciatura, un postgrado o todos ellos? El establecimiento de carreras técnicas o de pregrado siempre fue objeto de discusión a lo interno de las escuelas de ciencia política. Con el paso de los años la URL y la UFM optaron por no ofrecer carreras técnicas intermedias, sino únicamente licenciaturas, mientras que la USAC decidió ofrecer además de la licenciatura, también un grado técnico (de tres años). Como se discute más adelante, las tres universidades optaron por ofrecer también programas de maestría. Sin embargo, ninguna de las tres ofrece actualmente, o ha ofrecido en el pasado, programas de doctorado en ciencia política.

Otras disyuntivas se relacionan con el contenido de los programas de estudio. ¿Deben ofrecerse cursos en ciencia política mayoritariamente, o debe ser el programa de estudios más bien de carácter multidisciplinario? ¿Se debe enfatizar las capacidades de investigación o las de administración pública? Esas y otras preguntas similares han estado en la mesa de discusión de las distintas escuelas de ciencia política y los programas de estudio han reflejado y todavía reflejan, esas disyuntivas. Las escuelas de ciencia política de las tres universidades antes señaladas han optado por programas multidisciplinarios que van más allá de la ciencia política per se. Esto ha estado determinado en buena parte por el mercado laboral de los futuros egresados y la dinámica política del país. Dada la diversidad de posibles opciones laborales para los egresados de las carreras de ciencias políticas, los programas de estudio incluyen cursos de ciencia política, relaciones internacionales y sociología, pero también cursos en las áreas de derecho, economía e incluso administración pública. Con el paso de los años se han incorporado cursos de metodología de investigación, matemática y estadística.

Actualmente los programas de estudio en todas las universidades son todavía multidisciplinarios, siendo la ciencia política únicamente una de las ramas de estudio que conllevan a obtener una licenciatura. Es por ello que muchos de los docentes en las carreras de ciencia política no son 
necesariamente politólogos, sino que especialistas en otras ramas de las ciencias sociales. El currículo de estudios es generalmente cerrado: el estudiante debe cumplir con todos los cursos asignados, sin tener posibilidad de escoger entre diversas opciones.

En la URL inicialmente se ofrecían licenciaturas con especializaciones en relaciones internacionales, administración pública, sociología política y teoría política, pero en años recientes se decidió mantener únicamente la licenciatura en ciencias políticas. La carrera de trabajo social (técnico y licenciatura) fue trasladada a la Facultad de Ciencias Políticas y Sociales, al convertirse ésta de Instituto en Facultad. La USAC por su lado, estableció desde un inicio tres diferentes carreras dentro de la Escuela de Ciencia Política: la licenciatura en ciencia política, la licenciatura en relaciones internacionales y la licenciatura en sociología. La UFM puso mayor énfasis desde un inicio en el área de relaciones internacionales.

Cabe notar que en las tres universidades la mayoría de estudiantes se inclinan inicialmente por obtener alguna especialización en el área de relaciones internacionales, aunque ello está cambiando en años recientes ${ }^{12}$. El interés de los estudiantes en seguir una licenciatura en relaciones internacionales se asocia a las perspectivas que tienen inicialmente de entrar a formar parte del servicio diplomático. Sin embargo, éstas se han visto frustradas, ya que pocos egresados han sido absorbidos por el Ministerio de Relaciones Exteriores de Guatemala, en el cual no existe formalidad en la carrera diplomática; los nombramientos de personal de distinto nivel están generalmente sujetos a las decisiones de los gobiernos de turno ${ }^{13}$.

El perfil del egresado de las carreras en ciencia política ha ido cambiando conforme ha ido cambiando el contexto político del país. En un inicio existían dudas acerca del mercado del trabajo para los egresados de licenciaturas en ciencia política, relaciones internacionales o sociología, dado que eran carreras novedosas. La mayoría de estudiantes que ingresaban a los programas de licenciatura eran recién egresados del nivel secundario y el desafío era convertirlos en profesionales de una ciencia poco conocida en el medio. Aunque en un inicio fue difícil ubicar laboralmente a los egresados de estas áreas, al darse la apertura de espacios para la investigación de "lo político" se generó mayor demanda de egresados de ciencia política, tanto en centros de investigación y en las universidades mismas, así como en oficinas públicas, organizaciones no gubernamentales y organismos internacionales. Las perspectivas laborales mejoraron aún más luego de la firma de los acuerdos de paz, cuando muchos proyectos con apoyo internacional fueron establecidos en el país con el fin de poner en marcha los acuerdos.

Cabe indicar que a diferencia de otros países, en Guatemala la docencia como opción laboral es limitada, no sólo para los politólogos, sino para distintos profesionales. La universidad pública, USAC, es la única en donde existe un sistema de plaza permanente y relativamente bien remunerada, en la cual los politólogos pudieran desarrollar una carrera docente. Aunque en años recientes han hecho esfuerzos por mejorar las condiciones laborales de los profesores, en las universidades privadas todavía predomina un sistema de "catedrático-horario", en el cual profesionales que laboran en otra institución, llegan ciertas horas del día a dar una cátedra. También hay algunos

12 Entrevista con Renzo Rosal, ex decano de la Facultad de Ciencias Políticas y Sociales de la Universidad Rafael Landívar, abril, 2005.

13 Algunas administraciones de gobierno durante el período democrático han iniciado procesos de formalización de la carrera diplomática en conjunto con algunas universidades, pero no se ha logrado consolidar estos esfuerzos. 
a plazas de tiempo completo, pero en general no son plazas permanentes. En todo caso, en ninguna universidad existe un sistema equivalente al tenure-track. Aun en la universidad pública, las plazas no son necesariamente otorgadas con base en mérito académico.

Más allá del mercado laboral, desde la apertura democrática y en especial luego de la firma de los acuerdos de paz, varios politólogos han optado por tener columnas de opinión pública permanentes en diversos medios de comunicación, lo cual permite que desde una perspectiva más académica, se discutan ciertos temas coyunturales. La prensa en general, y aun la televisión y la radio, también han buscado en años recientes la opinión de los académicos en general. Los politólogos son buscados sobre todo en épocas pre-electorales, pero la mayoría son colaboraciones ad honorem.

En general la producción académica de los politólogos que viven en Guatemala es limitada, dado que muchos de los egresados se dedican a otras actividades y no a la investigación social ${ }^{14}$. La única publicación regular específica de Ciencia Política sigue siendo la de la Escuela de Ciencia Política de la USAC. Los politólogos de otras universidades tienden a publicar en las revistas multidisciplinarias de los centros de investigación como ASIES, FLACSO e INCEP.

Es importante mencionar un grupo meta distinto interesado en la ciencia política: los profesionales de otras disciplinas que ya trabajan en otras carreras y cuyas perspectivas laborales se ubican más bien en otros campos, y no en el de la ciencia política necesariamente. En buena parte por esa razón se establecieron programas de maestría. Las escuelas de ciencia política han ido variando las maestrías que ofrecen de conformidad con el contexto del país. La UFM estableció en 1997 la maestría en Relaciones Internacionales, la cual todavía ofrece. La Facultad de Ciencias Políticas y Sociales de la URL ha sido la que más variedad de maestrías ha ofrecido desde mediados de los años 90 , entre ellas una maestría en ciencias políticas, una en políticas públicas, una en transparencia y buen gobierno, una en estudios estratégicos y una en relaciones internacionales. En otras palabras, las maestrías hasta el momento, no han tenido en su mayoría al menos, el objetivo de formar investigadores o docentes, sino ofrecer la ciencia política como un complemento a la formación de profesionales de otras disciplinas, o como una especialización para los politólogos.

Otra disyuntiva de las escuelas de ciencia política ha sido hasta dónde debe llegar el alcance de sus programas de estudio. ¿Se debe enfatizar el estudio de la realidad política y social de Guatemala o más bien se debe enfatizar la comprensión de paradigmas y de realidades más amplias como la centroamericana, la latinoamericana o la del Tercer Mundo? En general se ha optado por poner énfasis en el estudio de la problemática política guatemalteca ${ }^{15}$. Esto difiere, por ejemplo, de los programas de estudio en EEUU, en donde la ciencia política comparada es un componente esencial tanto en los programas de pregrado como en los de postgrado.

Una última disyuntiva se relaciona con la proyección de la ciencia política hacia el resto de la población. ¿Se debe limitar la enseñanza de la disciplina a los estudiantes universitarios o deben las unidades académicas también brindar capacitación política a otros guatemaltecos? Algunas escuelas de ciencia política han querido trascender la enseñanza formal y han buscado dar capa-

14 Hay profesionales de diversas disciplinas que publican libros y artículos acerca de temas políticos, en particular abogados, algunos con maestrías en ciencias sociales en el extranjero.

15 Esta es una disyuntiva que enfrentan también otras escuelas de ciencia política en América Latina. Algunos estudiantes latinoamericanos comentan con frecuencia que es difícil tener una visión de conjunto de América Latina si se limitan a estudiar en sus países. Es más bien cuando llegan al extranjero a realizar estudios en que adquieren una visión más global. 
citación a personas que no necesariamente tienen posibilidad de seguir estudios universitarios. En parte por esa razón las escuelas de ciencia política han creado centros o institutos adscritos a su estructura, enfocándose en diversos temas ${ }^{16}$.

La Escuela de Ciencia Política de la USAC por su lado estableció el Instituto de Investigaciones Políticas y Sociales (IIPS), y la Facultad de Ciencias Políticas y Sociales de la URL estableció, también en los años 90, dos institutos: el Instituto de Transformación de Conflictos para la Construcción de la Paz (INTRAPAZ) y el Instituto de Gerencia Política (INGEP). La EPRI de la UFM estableció a finales de los años 90 el Centro para el Análisis de Decisiones Públicas (CADEP). Estos centros realizan actividades de investigación, pero algunos también dan capacitación en temas políticos a grupos especificos tales como miembros de partidos políticos o de organizaciones sociales.

\section{LOS VÍNCULOS CON OTRAS CIENCIAS SOCIALES}

Dado el carácter multidisciplinario de las escuelas de ciencia política, han existido fuertes vínculos con otras ciencias sociales y con el derecho, lo cual en general no ha sido positivo para el desarroIlo independiente de la disciplina.

Los egresados de las distintas carreras ofrecidas por las universidades guatemaltecas deben formar parte de un colegio profesional. Los egresados de licenciaturas en ciencia política, relaciones internacionales o sociología deben registrarse en el Colegio de Abogados y Notarios de Guatemala. El cercano vínculo entre la carrera de leyes y la de ciencia política tiene antecedentes en el país. Cabe indicar que antes de que surgiera la ciencia política en Guatemala, eran los abogados los encargados de estudiar y debatir temas políticos, especialmente en el enfoque formalista de la política que prevaleció durante mucho tiempo. Muchos de los directores de las escuelas de ciencia política a través de los años (y muchos de los catedráticos) han sido abogados ${ }^{17}$. Más aún, la Escuela de Ciencia Política de la USAC estuvo adscrita por varios años a la Facultad de Derecho.

Desde hace algunos años se vienen haciendo esfuerzos por formar un colegio profesional independiente del Colegio de Abogados. Se estuvo cerca de ello cuando la legislación guatemalteca requería de un mínimo de 100 egresados para formar un colegio profesional, pero la falta de consenso entre los egresados - debido en parte a las diferencias ideológicas existentes entre los egresados de las diferentes escuelas en ese momento- lo impidieron. La legislación requiere ahora un mínimo de 500 egresados, graduados o incorporados a universidades guatemaltecas, para formar un nuevo colegio profesional. En la actualidad, según el Directorio del Colegio de Abogados de Guatemala del año 2004, están registrados 82 internacionalistas (licenciados en relaciones internacionales), 53 politólogos (licenciados en ciencia política) y 66 sociólogos (licenciados en sociología). En el año 2004 se iniciaron reuniones específicas de los egresados de esas tres carreras para lograr mejores términos en la colegiación profesional en el Colegio de Abogados, ya que en la actualidad no se reciben mayores beneficios, pese a tener que cubrir las cuotas regulares de colegiación de los profesionales de las ciencias jurídicas ${ }^{18}$.

16 Estos programas han sido también promovidos por donantes internacionales, en especial después de los acuerdos de paz.

17 Aunque en mayor medida la conducción de las escuelas de ciencia política ha estado en años recientes en manos de politólogos, con frecuencia se nombra en el cargo de decano o director de ciencia política en algunas universidades a profesionales de otras disciplinas, en especial abogados y economistas.

18 Entrevista con Carlos Escobar Armas, ex director del Instituto de Ciencias Políticas de la URL, abril, 2005. 
El número de egresados de las distintas maestrías relacionadas con la ciencia política en Guatemala o en el extranjero es difícil de determinar, al igual que el de los graduados de pregrado o de doctorado en universidades del extranjero. No obstante, se sabe que alrededor de media docena de guatemaltecos han obtenido doctorados en ciencia política en el extranjero. La mayoría de ellos han optado por permanecer en el país, pero no necesariamente se han dedicado a la docencia. Algunos de ellos trabajan en universidades del extranjero ${ }^{19}$.

\section{PARADIGMAS METODOLÓGICOS PREVALECIENTES}

La ciencia política en Guatemala no sufre de las fracturas metodológicas por las que atraviesa, por ejemplo, la disciplina en Estados Unidos entre los proponentes de la ciencia política cualitativa versus los proponentes de la ciencia política cuantitativa -y en este último caso entre los cientistas políticos que se inclinan por los modelos formales (rational choice) o quienes se inclinan por los modelos estadísticos. Las fracturas, como se mencionó antes, son más bien de tipo ideológico.

La ciencia política en Guatemala tiene un marcado enfoque cualitativo, en el cual prevalece el estudio de la teoría política y la metodología cualitativa de investigación. El énfasis continúa estando en el estudio de instituciones políticas, aunque en años recientes se ha incursionado en otros campos. La UFM ha optado en años recientes por enfatizar el estudio de la opción pública (public choice).

Además del énfasis en lo cualitativo y en los autores latinoamericanos, el enfoque de la ciencia política en Guatemala se asocia más bien a la ciencia política de la Europa continental, en particular España y en menor medida Francia y Alemania. Ello se debe en parte a que hasta hace poco, quienes realizaban estudios de postgrado en ciencias sociales tendían a ir a estos países. Al regresar, impartían cátedras y realizaban investigaciones utilizando los modelos allí prevalecientes. En años recientes ha aumentado el número de profesionales que buscan obtener maestrías o doctorados en Estados Unidos o el Reino Unido, pero el idioma inglés es con frecuencia un impedimento. En esa misma línea de pensamiento, cabe resaltar que muy pocos de los múltiples textos relacionados con la ciencia política que se producen en EEUU o el Reino Unido son traducidos prontamente al castellano.

No obstante, sí existen algunos centros de investigación que aplican metodologías cuantitativas en sus investigaciones, en particular porque desde hace algunos años diversos donantes internacionales que apoyan proyectos de investigación en el área de ciencia política demandan mayor utilización de datos cuantificables. Entre estos puede contarse la serie de estudios acerca de la cultura política de los guatemaltecos, basado en encuestas de opinión pública ${ }^{20}$.

\section{LOS DESAFIOS DE LA CIENCIA POLITICA EN LA ACTUALIDAD}

La ciencia política coayudó en momentos cruciales de la historia política de Guatemala al logro de una transición del autoritarismo a la democracia. Como indica Thesing, las escuelas de ciencia

19 No existe un registro central ni obligación de colegiarse para los graduados del extranjero que no van a prestar sus servicios en oficinas públicas. Dado que la docencia como carrera no es una profesión bien remunerada, han optado por trabajar en organismos internacionales, centros de investigación, el gobierno o en ONG.

20 El más reciente de estos estudios es el VI estudio acerca de la cultura democrática de los guatemaltecos. Azpuru, Dinorah (2004). El primero de estos estudios fue realizado en 1993. 
política en la URL y la USAC contribuyeron decisivamente a que la violencia en Guatemala cediera ante una alternativa democrática que, entretanto, pudo imponerse en amplia medida ${ }^{21}$.

Si bien la ciencia política no enfrenta en la actualidad retos tan enormes como los que enfrentaba en sus inicios hace 35 años -incluyendo con frecuencia la misma sobrevivencia de los estudiosos de la disciplina-, sí enfrenta importantes desafíos.

El mayor desafío es cómo contribuir, desde la cátedra y la investigación y a través de los profesionales egresados de las distintas universidades, a la consolidación de una democracia todavía incipiente. Es común que académicos extranjeros consideren a Guatemala una de las democracias menos desarrolladas en el continente. Dicha visión pesimista ha tendido a mejorar desde la firma de los acuerdos de paz, pero todavía prevalece. ¿Por qué impera esta visión negativa en el extranjero? ¿Es producto de una realidad política existente o más bien son impresiones sin fundamentos empíricos? ¿En qué aspectos ha realmente avanzado la democracia guatemalteca vis-àvis el pasado y qué aspectos deben mejorarse para avanzar hacia una democracia consolidada? Estas son preguntas que pueden ser respondidas en mejor forma por politólogos guatemaltecos que por académicos extranjeros que a veces no conocen a fondo la realidad del país.

Más allá del diagnóstico acerca de la realidad política, la ciencia política también está llamada a proponer soluciones que lleven a Guatemala hacia la estabilidad democrática. Ello implica que además de los temas tradicionales de la ciencia política, ésta debe entre otros prestar atención a temas como la reforma, modernización y fortalecimiento institucional -en particular de instituciones democráticas básicas como los partidos políticos y el Congreso-; las políticas públicas eficaces contra la corrupción, la criminalidad y la violencia; la promoción de participación y derechos de los grupos marginados, la adaptación de la política a la realidad multiétnica del país, el desarrollo de políticas sociales efectivas, y la construcción y reforzamiento de la cultura democrática en el país.

Un aspecto positivo que puede coadyuvar a enfrentar con éxito estos desafíos es que parece existir hoy día mayor acercamiento entre las escuelas de ciencia política de las distintas universidades, que en cierta medida han superado las divisiones ideológicas que prevalecieron hasta hace pocos años y que han ido desapareciendo a raíz de la finalización del conflicto armado interno (y de la guerra fría a nivel internacional). Todavía prevalecen fracturas ideológicas entre las universidades, pero es de esperar que éstas se superen con el paso de los años.

El nuevo contexto internacional también requiere que la ciencia política guatemalteca trascienda el análisis de la realidad nacional y adquiera una visión más amplia, en especial por los retos que presenta la globalización. A este respecto, es positivo observar que los jóvenes estudiosos de la disciplina en Guatemala han buscado acercamientos con otros países latinoamericanos. En el año 2004 se estableció por primera vez el Capítulo Guatemala de la Asociación Latinoamericana de Estudiantes y Graduados de Ciencia Política22. En esa misma línea de apertura hacia otros países e ideas, Guatemala tomó el liderazgo en la organización del II Congreso Centroamericano de Ciencia Política, a realizarse en el país en octubre del año 2005²3. Todavía existe baja participa-

21 Thesing, Josef (2000).

22 La ALEGCIPOL fue creada en 1995.

23 Entrevista con Juan Luis Castilla, miembro del Comité Organizador del II Congreso Centroamericano de Ciencia Política, "Centroamérica, globalización y nuevas configuraciones del poder mundial". Abril, 2005. en la organización de este Congreso participan las diversas escuelas de Ciencia Política en Guatemala, así como los principales centros de investigación académica. 
ción de politólogos guatemaltecos en reuniones académicas de mayor envergadura, tales como la reunión bi-anual de Latin American Studies Association o la reunión anual de la American Political Science Association, aunque sí se presentan ponencias en algunos paneles de las mismas.

En conclusión, a diferencia de otras realidades en donde la ciencia política muchas veces se desarrolla en un nivel abstracto -y con frecuencia desligada de los hechos políticos-, la disciplina en Guatemala está llamada a una investigación de alto nivel científico, pero cuyos resultados puedan ser aplicados a una realidad concreta. La ciencia política es todavía una ciencia relativamente joven en Guatemala, que tiene el potencial de contribuir, aun más que otras disciplinas, a la construcción de una democracia estable y una paz firme y duradera.

Pero para lograr esos propósitos, la ciencia política tiene que adquirir mayor independencia como disciplina, desligándose de los fuertes vínculos que todavía mantiene con otras ciencias sociales y con el derecho. Debe también, convertirse en una ciencia política más moderna, que enfatice el estudio de paradigmas más globales de la politología. Metodológicamente, la formación de los nuevos egresados debe tener mayor énfasis cuantitativo, brindándole al estudiante los instrumentos que le permitan analizar una problemática desde diversas perspectivas metodológicas. Finalmente, si bien el estudio de los problemas de Guatemala es fundamental, es difícil comprender la realidad guatemalteca si no se trascienden las fronteras de lo local, es por ello que las fronteras geográficas de estudio deben ampliarse, utilizando en particular la ciencia política comparada.

\section{REFERENCIAS}

Azpuru, Dinorah. 2004. La Cultura Política de la Democracia en Guatemala. Guatemala: ASIES/Nanderbilt University/USAID/ARD. Colegio de Abogados y Notarios de Guatemala. 2004. Directorio 2004 del Colegio de Abogados y Notarios de Guatemala. Guatemala.

Kelly, Philip. 1998. Assessing Democracy in Latin America: A Tribute to Russell H. Fitzgibbon. Boulder, Co.: Westview Press. Linz, Juan y Alfred Stepan. 1996. Problems of Democratic Transition and Consolidation, Southern Europe, South America and Post-Communist Europe. Baltimore and London: The Johns Hopkins University Press.

Marsh, David y Gerry Stroker. 2002. Theory and Methods in Political Science. Great Britain: Palgrave McMillan.

Pásara, Luis. 2003. Ilusión y Cambio en Guatemala: El proceso de paz, sus actores, logros y límites. Guatemala: Universidad Rafael Landivar, Instituto de Investigaciones Jurídicas.

Thesing, Josef. 2000. Democracia en Guatemala: experiencias y perspectivas. Discurso pronunciado en el Acto de Celebración del 30 Aniversario de la Facultad de Ciencias Políticas y Sociales de la Universidad Rafael Landívar el 4 de abril del 2000. Guatemala: Publicado por el Instituto Centroamericano de Estudios Políticos.

\footnotetext{
Dinorah Azpuru es Doctora (Phd) y Master en Ciencia Política por la Universidad de Pittsburgh, Estados Unidos. Actualmente es profesora de Ciencia Política en Wichita State University (WSU), Estados Unidos. Además, es Miembro Asociado de la Asociación de Investigación y Estudios Sociales (ASIES) en Guatemala. Ha sido profesora en la Universidad de Vanderbilt (EEUU) y la Universidad Rafael Landívar en Guatemala, en donde también fue Vicedecana de la Facultad de Ciencias Políticas y Sociales y Directora del Departamento de Ciencias Políticas. Ha publicado extensamente acerca de la cultura democrática en Guatemala, los procesos de paz y democratización en Centroamérica, con particular énfasis en Guatemala.

(E-mail: dazpuru@yahoo.com)
} 